\title{
Tumour cells co-opt synaptic signalling
}

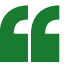 \\ treatment of xenografted mice with an AMPAR antagonist reduced glioma cell proliferation}

Gliomas and breast-cancer-derived brain metastases (B2BMs) are types of brain tumour associated with poor prognosis. Whether and how the cells in these tumours interact synaptically with other brain cells has not been clear. Three papers in Nature now detail synaptic interactions between neurons and glioma or B2BM cells that seem to promote tumour growth.

Venkatesh et al. and Venkataramani et al. both examined the ultrastructure of glioma cells using electron microscopy and noticed synaptic structures involving presynaptic neurons and tumour cell postsynapses. Gene-expression analysis revealed that glioma cells express many synaptic genes, including the gene that encodes GLUA2, a subunit of AMPA receptors (AMPARs), which are a family of ionotropic glutamate receptors.

Both groups recorded excitatory postsynaptic potentials (EPSCs)

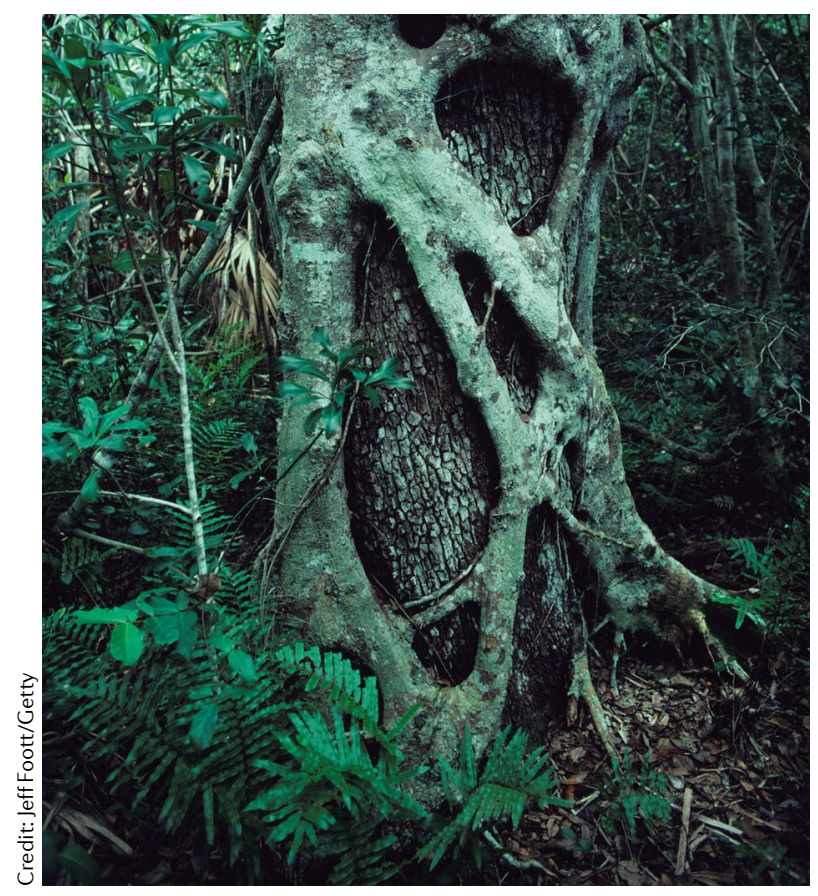

in glioma cells in tumour samples from human patients or in slices of tumour-xenografted mouse brains. These EPSCs depended on neuronal activity, suggesting that the observed neurogliomal synapses were functional. Moreover, in line with the gene-expression data, the EPSCs depended on $\mathrm{Ca}^{2+}$-permeable AMPARs.

Further electrophysiological recordings and calcium imaging revealed that some glioma cells exhibited prolonged inward currents that relied, in part, on neuronal-activity-induced increases in extracellular potassium. Previous work has shown that glioma cells are connected by gap junctions located at cellular processes called tumour microtubes. Venkatesh et al. and Venkataramani et al. observed synchronized patterns of calcium activity in connected glioma cells, suggesting that calcium waves might spread through gap junction-linked glioma cell networks.

To determine the functional effects of the $\mathrm{Ca}^{2+}$-permeable AMPARs on the glioma cells, both groups xenografted glioma cells expressing either wild-type GLUA2 or a dominant-negative form of GLUA2 (GLUA2-DN) into mouse brains. Mice bearing GLUA2-DN-expressing gliomas had less-invasive tumours, had a smaller tumour burden and survived longer than did mice with wild-type gliomas. Furthermore, treatment of xenografted mice with an AMPAR antagonist reduced glioma cell proliferation. Thus, glioma cell AMPAR activation promotes tumour growth.

In a complementary study, Zeng et al. examined published B2BM transcriptomic data and found that these tumours often express genes encoding components of metabotropic glutamate receptors called NMDA receptors (NMDARs) - particularly the subunit GLUN2B. Phosphorylated GLUN2B (pGLUN2B) - which is crucial for activation of cell-surface NMDARs - was identified in B2BMs from patients, often at higher levels than in their primary breast tumours. Breast-cancer lines selected for their proficiency in causing brain metastases showed high expression of pGLUN2B and other NMDAR subunits, as well as NMDAR-mediated currents and calcium transients in response to glutamate or NMDA. Using super-resolution microscopy of brain metastases, the authors observed co-localization of labelled presynapses, pGLUN2B and B2BM cells. B2BM cells seemed to be positioned alongside the synaptic cleft between two neurons, much like astrocytes in a tripartite synapse. Compared with wild-type B2BM cells, B2BM cells in which GLUN2B was knocked down seeded brain metastases in mice just as proficiently, but led to a lower brain-tumour burden and longer survival, suggesting that NMDAR signalling in brain metastases may promote brain metastatic growth.

Together, these results describe how certain types of tumour can co-opt glutamate signalling in the brain to drive tumour growth.

Natasha Bray, Senior Editor, Nature Reviews Neuroscience This article is modified from the original in Nat. Rev. Neurosci. (https://doi.org/10.1038/ s41583-019-0230-5).

ORIGINAL ARTICLES Venkatesh, H. S. et al. Electrical and synaptic integration of glioma into neural circuits. Nature 573, 539-545 (2019) | Venkataramani, V. et al. Glutamatergic synaptic input to glioma cells drives brain tumour progression. Nature 573, 532-538 (2019)| Zeng, Q. et al. Synaptic proximity enables NMDAR signalling to promote brain metastasis. Nature 573, 526-531 (2019) 